\title{
CYCLIC MAPS IN RATIONAL HOMOTOPY THEORY
}

\author{
GREGORY LUPTON AND SAMUEL BRUCE SMITH
}

\begin{abstract}
The notion of a cyclic map $g: A \rightarrow X$ is a natural generalization of a Gottlieb element in $\pi_{n}(X)$. We investigate cyclic maps from a rational homotopy theory point of view. We show a number of results for rationalized cyclic maps which generalize well-known results on the rationalized Gottlieb groups.
\end{abstract}

\section{INTRODUCTION}

Let $X$ be a space with basepoint $x_{0}$. Denote by $\operatorname{map}(X, X ; 1)$ the component of the identity in the unbased function space of maps from $X$ to itself, and by $\omega: \operatorname{map}(X, X ; 1) \rightarrow X$ the evaluation map defined by $\omega(f)(x)=f\left(x_{0}\right)$. In [Got69], Gottlieb introduced and studied the evaluation subgroups

$$
G_{n}(X)=\omega_{\#}\left(\pi_{n}(\operatorname{map}(X, X ; 1))\right) \subseteq \pi_{n}(X) .
$$

Note that $G_{n}(X)$ can alternatively be described as homotopy classes of maps $f: S^{n} \rightarrow X$ such that $(f \mid 1): S^{n} \vee X \rightarrow X$ admits an extension $F: S^{n} \times X \rightarrow X$ up to homotopy. These so-called Gottlieb groups admit a number of generalizations. One such was introduced and studied by Varadarajan. In [Var69], he defines the homotopy set $G(A, X)$ of cyclic maps from $A$ to $X$, that is, homotopy classes of maps $f: A \rightarrow X$ such that $(f \mid 1): A \vee X \rightarrow X$ admits an extension $F: A \times X \rightarrow X$. The Gottlieb group occurs when $A=S^{n}$. Note that the set of cyclic maps $G(A, X)$ can alternatively be described as the set of homotopy classes of maps from $A$ to $X$ that admit a lift through the evaluation map $\omega: \operatorname{map}(X, X ; 1) \rightarrow X$.

The rationalized Gottlieb groups $G_{n}(X) \otimes \mathbb{Q}$ have been studied extensively (see [FH82, Tan83, Opr95]). Our purpose in this paper is to study rationalized cyclic maps with an eye toward obtaining natural generalizations of known results. In [Got69] Gottlieb studied the question of when the Gottlieb group of $X$ is contained in the kernel of the mod $p$ or rational Hurewicz homomorphisms. Let $h_{\infty}: \pi_{*}(X) \rightarrow$ $H_{*}(X ; \mathbb{Q})$ denote the rational Hurewicz homomorphism. Then Gottlieb's main results for the rational case can be summarized as follows:

Theorem 1.1 ([Got69, Th.4.1,Th.5.1]). Let $X$ be a space with finitely generated homology. Then $G_{2 n}(X) \subseteq \operatorname{ker}\left(h_{\infty}\right)$ and if the Euler characteristic $\chi(X) \neq 0$ then $G_{2 n-1}(X) \subseteq \operatorname{ker}\left(h_{\infty}\right)$.

Gottlieb also showed that $G_{2}(X)$ is a torsion group - and hence that $G_{2}(X) \otimes \mathbb{Q}=$ 0 -in the case that $X$ has a finite number of non-zero rational homology groups [Got69, Th.7.1].

Date: July 10, 2018

2000 Mathematics Subject Classification. 55P62, 55Q05.

Key words and phrases. Evaluation map, Gottlieb group, function space, cyclic map, rational homotopy, minimal models. 
Félix and Halperin significantly sharpened these results. Let $X$ be a simply connected space. Then the rational category of $X$ - denoted by $\operatorname{cat}_{0}(X)$ - may be defined as the Lusternik-Schnirelmann category of the rationalization of $X$, that is, $\operatorname{cat}_{0}(X)=\operatorname{cat}\left(X_{\mathbb{Q}}\right)$. Further, the rationalized Gottlieb group of $X$ is contained in the Gottlieb group of the rationalization of $X$, or $G_{n}(X) \otimes \mathbb{Q} \subseteq G_{n}\left(X_{\mathbb{Q}}\right)$ (these agree if $X$ is finite [Lan75]).

Theorem 1.2 ([FH82, Th.III]). Let $X$ be a simply connected space of finite rational category. Then $G_{2 n}\left(X_{\mathbb{Q}}\right)=0$ and $\sum_{n} \operatorname{dim}\left(G_{2 n+1}\left(X_{\mathbb{Q}}\right)\right) \leq \operatorname{cat}_{0}(X)$.

In this paper, our main results are presented in Section 3. There, we give a generalization to cyclic maps of the Félix-Halperin result on even dimensional Gottlieb groups. Corollary 3.4 shows that cyclic maps of rational spaces are trivial under certain conditions. That result includes the part of Theorem 1.2 about trivial even dimensional Gottlieb groups of a rational space as a special case.

We also generalize Gottlieb's result about odd dimensional Gottlieb groups, as follows: To say that $\alpha \in \pi_{n}(X)$ is in the kernel of the rational Hurewicz homomorphism is simply to say that $H_{*}(\alpha)=0: H_{*}\left(S^{n} ; \mathbb{Q}\right) \rightarrow H_{*}(X ; \mathbb{Q})$. More generally, write $S H_{*}(X ; \mathbb{Q})$ for the rational spherical homology of $X$ : that is, $S H_{*}(X ; \mathbb{Q})$ is the image of the rationalized Hurewicz homomorphism $h_{\mathbb{Q}}: \pi_{*}(X) \otimes \mathbb{Q} \rightarrow H_{*}(X ; \mathbb{Q})$. Note that a map $f: A \rightarrow X$ induces a map $S H_{*}(f): S H_{*}(A ; \mathbb{Q}) \rightarrow S H_{*}(X ; \mathbb{Q})$. We give a vanishing result for the map induced on rational spherical homology by a cyclic map in Corollary 3.10, which extends Gottlieb's odd dimensional result.

Also in Section 3, we show results about the homomorphism induced on rational homotopy groups by a cyclic map. One such result is Corollary 3.7, which gives a bound on the size of the image that a cyclic map may induce on odd dimensional rational homotopy groups. It complements the Félix-Halperin result on odd dimensional Gottlieb groups. Many of the results in Section 3 are actually proved for a more general class of maps than cyclic maps, namely, maps that factor through an $H$-space.

In Section 4 we give a number of examples of cyclic maps between rational spaces to illustrate our results in Section 3. We also give some sample computations of the rationalization of the set of cyclic maps.

All spaces in the sequel are assumed to be simply connected CW complexes with rational homology of finite type - with the exception of mapping spaces such as $\operatorname{map}\left(X, X ; 1_{X}\right)$. We denote the set of homotopy classes of maps from $X$ to $Y$ by $[X, Y]$. We often do not distinguish between a map and the homotopy class it represents. If $f: A \rightarrow B$ is a map, then $f^{*}$ denotes pre-composition by $f$ and $f_{*}$ denotes post-composition by $f$. Thus we obtain maps of homotopy sets $f^{*}:[B, X] \rightarrow[A, X]$ and $f_{*}:[X, A] \rightarrow[X, B]$. We use $H_{*}(f)$ and $H^{*}(f)$ to denote the map induced on homology, respectively cohomology, by the map of spaces $f$, and $f_{\#}$ to denote the map induced on homotopy groups. We denote the rationalization of a space $X$ by $X_{\mathbb{Q}}$ and of a map $f$ by $f_{\mathbb{Q}}($ cf. [HMR75]).

We assume some familiarity with rational homotopy theory as introduced by Sullivan. Our main reference for this material is [FHT01]. The basic facts that we use are as follows: Each space $X$ has a unique Sullivan minimal model $\left(\mathcal{M}_{X}, d_{X}\right)$ in the category of simply connected DG (differential graded) algebras over $\mathbb{Q}$. This DG algebra $\left(\mathcal{M}_{X}, d_{X}\right)$ is of the form $\mathcal{M}_{X}=\Lambda V$, a free graded commutative algebra generated by a positively graded vector space $V$ of finite type. The differential $d_{X}$ is decomposable, in that $d_{X}(V) \subseteq \Lambda^{\geq 2} V$. A map $f: X \rightarrow Y$ has a Sullivan minimal 
model which is a DG algebra map $\mathcal{M}_{f}: \mathcal{M}_{Y} \rightarrow \mathcal{M}_{X}$. The Sullivan minimal model is a complete rational homotopy invariant for a space or a map. Since the minimal model is determined by the rational homotopy type, the minimal models of $X_{\mathbb{Q}}$ and $X$, and more generally those of $f_{\mathbb{Q}}$ and $f$, agree. There is a notion of homotopy for maps of Sullivan minimal models, which we refer to as DG homotopy (of maps of Sullivan minimal models). If $f, g: X \rightarrow Y$ are maps of rational spaces, then $f$ and $g$ are homotopic if and only if their Sullivan minimal models $\mathcal{M}_{f}$ and $\mathcal{M}_{g}$ are DG homotopic. Rational cohomology is readily retrieved from Sullivan minimal models: We have a natural isomorphism $H\left(\mathcal{M}_{X}, d_{X}\right) \cong H^{*}(X ; \mathbb{Q})$ and this isomorphism identifies $H\left(\mathcal{M}_{f}\right): H\left(\mathcal{M}_{Y}\right) \rightarrow H\left(\mathcal{M}_{X}\right)$ with $H^{*}(f): H^{*}(Y ; \mathbb{Q}) \rightarrow$ $H^{*}(X ; \mathbb{Q})$. Rational homotopy is retrieved as follows: Let $Q\left(\mathcal{M}_{X}\right) \cong V$ be the (quotient) module of indecomposables of $\mathcal{M}_{X}$. There is a natural isomorphism $Q\left(\mathcal{M}_{X}\right) \cong \operatorname{Hom}\left(\pi_{*}(X) \otimes \mathbb{Q}, \mathbb{Q}\right)$, that identifies $Q\left(\mathcal{M}_{f}\right): Q\left(\mathcal{M}_{Y}\right) \rightarrow Q\left(\mathcal{M}_{X}\right)$ with $\left(f_{\#} \otimes \mathbb{Q}\right)^{*}: \operatorname{Hom}\left(\pi_{*}(Y) \otimes \mathbb{Q}, \mathbb{Q}\right) \rightarrow \operatorname{Hom}\left(\pi_{*}(X) \otimes \mathbb{Q}, \mathbb{Q}\right)$.

For facts that we have used about minimal models beyond these basics, we have given specific references from [FHT01]. We use the standard notation and terminology for minimal models as used in [FHT01]. A map of DG algebras is called a quasi-isomorphism if it induces an isomorphism on cohomology. We mention that there is a discussion of Gottlieb groups, including a proof of Theorem 1.2 cited above, given in [FHT01, sec.28(d)].

\section{Generalities on Cyclic Maps}

In this section, we record some basic facts about the set $G(A, X)$. All of these results are either known as stated or occur as easy extensions of known results on cyclic maps or evaluation subgroups. The first is a useful result from [Var69].

Theorem 2.1 ([Var69, Th.1.3]). Let $\theta: B \rightarrow A$ be any map. Then $\theta^{*}:[A, X] \rightarrow$ $[B, X]$ restricts to a map of sets of cyclic maps $\theta^{*}: G(A, X) \rightarrow G(B, X)$.

Proof. Suppose that $F: A \times X \rightarrow X$ extends $(g \mid 1)$. Then $G: B \times X \rightarrow X$ defined by $G(b, x)=F(\theta(b), x)$ extends $(g \circ \theta \mid 1)$.

We obtain the following two immediate consequences of this.

Corollary 2.2. A cyclic map $g \in G(A, X)$ satisfies

$$
g_{\sharp}\left(\pi_{n}(A)\right) \subseteq G_{n}(X) .
$$

Proof. Take $\theta: S^{n} \rightarrow A$. Then $g_{\#}(\theta)=g \circ \theta=\theta^{*}(g): S^{n} \rightarrow X$ is in $G_{n}(X)$.

Corollary 2.3. $G(X, X)$ contains a self-equivalence of $X$ if and only if $X$ is an $H$-space.

Proof. Let $g: X \rightarrow X$ be a cyclic self-equivalence and $h: X \rightarrow X$ its homotopy inverse. Then, by Theorem 2.1, $1_{X} \simeq g \circ h$ is cyclic as well. The map $F: X \times X \rightarrow X$ that extends $1_{X} \vee 1_{X}$ is the needed multiplication.

Next we give some results, and establish our notation, concerning localization (see [HMR75] for full details). For any set of primes $P$, the $P$-localization map $e_{X}: X \rightarrow X_{P}$ induces a map of homotopy sets $\left(e_{X}\right)_{*}:[A, X] \rightarrow\left[A, X_{P}\right]$. Likewise, we have an induced map $\left(e_{A}\right)^{*}:\left[A_{P}, X_{P}\right] \rightarrow\left[A, X_{P}\right]$, which is a bijection of sets.

Lemma 2.4. Let $A$ and $X$ be simply connected and let $P$ be any set of primes. 
(1) $\left(e_{X}\right)_{*}$ restricts to a map $\left(e_{X}\right)_{*}: G(A, X) \rightarrow G\left(A, X_{P}\right)$.

(2) The bijection $\left(e_{A}\right)^{*}$ restricts to a bijection $\left(e_{A}\right)^{*}: G\left(A_{P}, X_{P}\right) \rightarrow G\left(A, X_{P}\right)$.

Proof. Both parts follow easily from standard properties of localization. Suppose that $f: A \rightarrow X$ is a cyclic map with affiliated map $F: A \times X \rightarrow X$. Then an affiliated map for $\left(e_{X}\right)_{*}(f)$ is given by $F_{P} \circ\left(e_{A} \times 1\right): A \times X_{P} \rightarrow X_{P}$. This shows (1). For (2), suppose $\alpha: A_{P} \rightarrow X_{P}$ is a cyclic map. Then $\left(e_{A}\right)^{*}(\alpha) \in G\left(A, X_{P}\right)$ by Theorem 2.1. Since the restriction of an injection is injective, it only remains to show that $\left(e_{A}\right)^{*}$ restricts to a surjection. For this, suppose $\beta \in G\left(A, X_{P}\right)$ has affiliated map $B: A \times X_{P} \rightarrow X_{P}$. Then $\beta=\left(e_{A}\right)^{*}\left(\beta_{P}\right)$, and $B_{P}: A_{P} \times X_{P} \rightarrow X_{P}$ is an affiliated map for $\beta_{P}$. The latter assertion uses uniqueness of localized maps, and the fact that both $e_{A} \vee 1: A \vee X_{P} \rightarrow A_{P} \vee X_{P}$ and $e_{A} \times 1: A \times X_{P} \rightarrow A_{P} \times X_{P}$ are $P$-localization maps.

Because we use minimal model techniques, we obtain results about homotopy classes of cyclic maps $G\left(A_{\mathbb{Q}}, X_{\mathbb{Q}}\right)$, or more generally about homotopy classes of $\operatorname{maps}\left[A_{\mathbb{Q}}, X_{\mathbb{Q}}\right]$.

Definition 2.5. Let $f: A \rightarrow X$ be a map of simply connected spaces. We say $f$ is a rationally cyclic map if its rationalization $f_{\mathbb{Q}}: A_{\mathbb{Q}} \rightarrow X_{\mathbb{Q}}$ is a cyclic map. More generally, we say $f$ is a $P$-local cyclic map if $f_{P}: A_{P} \rightarrow X_{P}$ is cyclic. We denote the set of homotopy classes of $P$-local cyclic maps from $A$ to $X$ by $G_{P}(A, X)$. From Lemma 2.4, we have $G(A, X) \subseteq G_{P}(A, X)$ for any set of primes $P$.

In general the inclusion $\left(e_{X}\right)_{*}(G(A, X)) \subseteq G\left(A, X_{P}\right)$ may be strict: See [FHT01, pp.378-380] for an (infinite) example with $\left(e_{X}\right)_{*}\left(G_{n}(X)\right) \subsetneq G_{n}\left(X_{\mathbb{Q}}\right)$, but note that $\left(e_{X}\right)_{*}\left(G_{n}(X)\right)=G_{n}\left(X_{P}\right)$ if $X$ is finite, by [Lan75]. We do have the following result, however.

Lemma 2.6. Let $A$ and $X$ be simply connected with $A$ a finite $C W$ complex. Then $P$-localization induces a finite-to-one map

$$
\left(\left(e_{A}\right)^{*}\right)^{-1} \circ\left(e_{X}\right)_{*}: G_{P}(A, X) \rightarrow G\left(A_{P}, X_{P}\right) .
$$

In particular, if $A$ is finite and $G\left(A_{\mathbb{Q}}, X_{\mathbb{Q}}\right)$ is trivial, that is, consists of a single element, then the set of rationally cyclic maps $G_{\mathbb{Q}}(A, X)$ is finite.

Proof. The finite-to-one assertion follows from [HMR75, Cor.5.4].

\section{Maps that Factor Through an $H$-Space}

In this section we show that being cyclic entails strong restrictions on a map. Most of our results flow from the following observation. Suppose that $f: A \rightarrow X$ is a cyclic map. As we remarked in the introduction, $f$ admits a lift through the evaluation map as follows

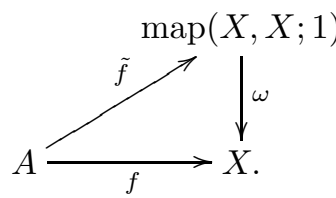

Now the identity component $\operatorname{map}(X, X ; 1)$ is an $H$-space, and thus a cyclic map factors through an $H$-space. Indeed, since we are assuming $A$ is simply connected, 
we can lift $\tilde{f}$ through the universal cover of $\operatorname{map}(X, X ; 1)$, which is again an $H$ space, and is also simply connected. Thus a cyclic map factors through a simply connected $H$-space. Such a factorization entails numerous consequences for a cyclic map. In fact, some of our results can be proved simply by assuming that the map $f: A \rightarrow X$ factors through some $H$-space - not necessarily the universal cover of $\operatorname{map}(X, X ; 1)$. This hypothesis on a map is definitely weaker than assuming the map to be cyclic. For instance, consider any map $f: S^{3} \rightarrow S^{3} \vee S^{3}$ that is not trivial on homotopy. Since $G_{3}\left(S^{3} \vee S^{3}\right)=0$, such a map is not a cyclic map. Since $S^{3}$ itself is an $H$-space, any such map certainly factors through an $H$-space.

Our primary interest is in drawing conclusions about cyclic maps. However, since our methods are those of rational homotopy, we need only require that the map be a rationally cyclic map as defined in Definition 2.5. Likewise, although a cyclic map actually factors through an $H$-space, we only require such a factorization after rationalization. In what follows, we will state our results so as to place as weak a hypothesis as possible on the maps and spaces. We remind the reader that our spaces are assumed to be simply connected with rational homology of finite type.

Our first consequence of these observations is as follows.

Proposition 3.1. Let $f: A \rightarrow X$ be a map whose rationalization factors through an $H$-space. Up to DG homotopy, the Sullivan minimal model $\mathcal{M}_{f}: \mathcal{M}_{X} \rightarrow \mathcal{M}_{A}$ of $f$ has image contained in the cycles of $\mathcal{M}_{A}$.

Proof. Suppose we have a factorization

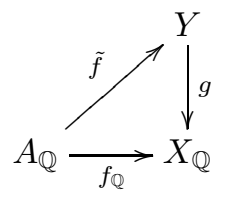

with $Y$ an $H$-space. Passing to minimal models, we have $\mathcal{M}_{f} \sim \mathcal{M}_{\tilde{f}} \circ \mathcal{M}_{g}: \mathcal{M}_{X} \rightarrow$ $\mathcal{M}_{A}$. Now the minimal model of an $H$-space has trivial differential, that is, $d_{Y}=0$ [FHT01, p.143]. For every element $\chi \in \mathcal{M}_{X}$, therefore, we have that $\mathcal{M}_{g}(\chi)$, and hence $\mathcal{M}_{\tilde{f}} \circ \mathcal{M}_{g}(\chi)$, is a cycle.

Proposition 3.1 gives a serendipitious justification of the terminology "cyclic map," at least in rational homotopy (cf. the remarks on nomenclature in [Var69]). With additional hypotheses on either $A$ or $X$, we can draw much stronger conclusions.

Theorem 3.2. Let $f: A \rightarrow X$ be a map whose rationalization factors through an $H$-space. If $X$ is of finite rational category and $H^{\text {odd }}(A ; \mathbb{Q})=0$, then $f$ is rationally trivial.

Proof. We prove the result by showing that the minimal model $\mathcal{M}_{f}: \mathcal{M}_{X} \rightarrow \mathcal{M}_{A}$ of $f$ is DG homotopic to the trivial map. See [FHT01, Sec.12(b)] for details about DG homotopy.

Suppose we have a factorization as in (1), with $Y$ an $H$-space. First we show that, up to DG homotopy, we can assume that $\mathcal{M}_{\tilde{f}}$ is zero on all odd-degree generators of $\mathcal{M}_{Y}$. For suppose that $\mathcal{M}_{Y}=\Lambda\left(\left\{a_{i}\right\}_{i \in I},\left\{b_{j}\right\}_{j \in J}\right)$, with each $a_{i}$ an even-degree generator and each $b_{j}$ an odd-degree generator. Recall that the differential $d_{Y}$ is zero, and so every element in $\mathcal{M}_{Y}$ is a cycle. Since $\tilde{f}\left(b_{i}\right)$ is an odd-degree cycle 
in $\mathcal{M}_{A}$, and since we are assuming that $H^{\text {odd }}(A ; \mathbb{Q})=0$, we have $\tilde{f}\left(b_{j}\right)=d_{A}\left(\eta_{j}\right)$ for some $\eta_{j} \in \mathcal{M}_{A}$. Now define a map $\Phi: \mathcal{M}_{Y} \rightarrow \mathcal{M}_{A} \otimes \Lambda(t, d t)$ on generators by setting $\Phi\left(b_{j}\right)=\tilde{f}\left(b_{j}\right) \otimes(1-t)+\eta_{j} \otimes d t, \Phi\left(a_{i}\right)=\tilde{f}\left(a_{i}\right) \otimes 1$. As the differential in $\mathcal{M}_{Y}$ is trivial, this map extends to a DG homotopy that starts at $\tilde{f}$ and ends at a map that is zero on each odd-degree generator $b_{j}$. So now assume that $\mathcal{M}_{\tilde{f}}$ is zero on odd-degree generators.

Let $\mathcal{I}$ be the ideal of $\mathcal{M}_{Y}$ generated by the odd-degree generators. We now show that $\mathcal{M}_{g}$ has image contained in $\mathcal{I}$. Clearly, for any odd-degree generator $x$ of $\mathcal{M}_{X}$, we must have $\mathcal{M}_{g}(x) \in \mathcal{I}$. To show that $\mathcal{M}_{g}(x) \in \mathcal{I}$ for $x$ an even-degree generator of $\mathcal{M}_{X}$, we argue inductively over degree. Assume that $\mathcal{M}_{g}(y) \in \mathcal{I}$, for all generators $y$ of degree $\leq 2 n-1$, and that $x$ is of degree $2 n$. Since $X$ has finite rational category, there exists an element $\eta \in \mathcal{M}_{X}$ such that $d_{X}(\eta)=x^{k}+R$, for some $k \geq 2$ and some $R$ in the ideal of $\mathcal{M}_{X}$ generated by generators of degree $\leq 2 n-1$. We justify this assertion in Lemma 3.3 below. Now write $\mathcal{M}_{g}(x)=P+Q$, with $Q \in \mathcal{I}$ and $P$ an element of the subalgebra of $\mathcal{M}_{Y}$ generated by the even-degree generators. Since the differential in $\mathcal{M}_{Y}$ is trivial, we have $\mathcal{M}_{g}\left(d_{X}(\eta)\right)=d_{Y}\left(\mathcal{M}_{g}(\eta)\right)=0$. Hence, $0=\mathcal{M}_{g}\left(x^{k}+R\right)=\left(\mathcal{M}_{g}(x)\right)^{k}+\mathcal{M}_{g}(R)=(P+Q)^{k}+\mathcal{M}_{g}(R)$. Since $Q \in \mathcal{I}$, and by our inductive assumption $\mathcal{M}_{g}(R) \in \mathcal{I}$, it follows that $P^{k} \in \mathcal{I}$ and hence that $P=0$. This completes the inductive step. We can start the induction with $n=1$, where the induction hypothesis is satisfied trivially. We have shown that $\mathcal{M}_{g}$ has image contained in $\mathcal{I}$. Since $\tilde{f}$ is zero on odd-degree generators, we have $\mathcal{M}_{\tilde{f}} \circ \mathcal{M}_{g}=0$.

The technical fact about minimal models used in the preceding proof is wellknown amongst experts in the field. We give a statement and proof of this useful fact for the sake of completeness.

Lemma 3.3. Let $X$ be a space of finite rational category. If $x \in \mathcal{M}_{X}$ is a generator of even degree $2 n$, then for some $k \geq 2$ there is an element $\eta \in \mathcal{M}_{X}$ such that $d_{X}(\eta)=x^{k}+R$, with $R$ in the ideal of $\mathcal{M}_{X}$ generated by generators of degree $\leq 2 n-1$.

Proof. First notice that if the even-degree generator is a cycle, $d_{X}(x)=0$, then the result is easily proved. For the assumption of finite rational category implies, in particular, that $X$ has finite rational cup length. Hence in this case, there is some $\eta$ such that $d_{X}(\eta)=x^{k}$ with $k \geq 2$. For the general case, in which $x$ is not a cycle, we use the mapping theorem of Félix-Halperin [FHT01, Th.29.5]. Suppose $x$ is of degree $2 n$, and let $\mathcal{M}_{X}\langle 2 n\rangle$ denote the quotient DG algebra obtained by factoring out the DG ideal of $\mathcal{M}_{X}$ generated by all generators of degree $\leq 2 n-1$. Let $\overline{d_{X}}$ denote the differential induced on the quotient by $d_{X}$. Then $\left(\mathcal{M}_{X}\langle 2 n\rangle, \overline{d_{X}}\right)$ is a minimal DG algebra and the projection $\mathcal{M}_{X} \rightarrow \mathcal{M}_{X}\langle 2 n\rangle$ induces a surjection of the modules of indecomposables. It follows from the mapping theorem that $\left(\mathcal{M}_{X}\langle 2 n\rangle, \overline{d_{X}}\right)$ also has finite rational category. By the argument at the start of this proof, there is some element $\eta \in \mathcal{M}_{X}\langle 2 n\rangle$ such that $\overline{d_{X}}(\eta)=x^{k}$ in $\mathcal{M}_{X}\langle 2 n\rangle$. Therefore, $d_{X}(\eta)=x^{k}+R$ for some $R$ in the ideal of $\mathcal{M}_{X}$ generated by generators of degree $\leq 2 n-1$, as asserted.

Corollary 3.4 (to Theorem 3.2). If $\operatorname{cat}_{0}(X)<\infty$ and $H^{\text {odd }}(A ; \mathbb{Q})=0$, then $G\left(A_{\mathbb{Q}}, X_{\mathbb{Q}}\right)$ is trivial. If, further, $A$ is a finite $C W$ complex, then $G_{\mathbb{Q}}(A, X)$, and hence $G(A, X)$, is a finite set. 
Proof. As we observed above, any cyclic map-including those in $G\left(A_{\mathbb{Q}}, X_{\mathbb{Q}}\right)$ factors through an $H$-space. Now apply Theorem 3.2 and Lemma 2.6.

Corollary 3.4 is a most satisfactory generalization to cyclic maps of the FélixHalperin result about even dimensional rational Gottlieb groups. Unfortunately, we have not found such a satisfactory generalization of their odd dimensional result. Our next three results take a step in this direction, however, giving restrictions on the homomorphism induced on homotopy groups by a cyclic map.

Proposition 3.5 (cf. [FHT01, Cor. on p.379]). Let $f: A \rightarrow X$ be a rationally cyclic map, with $X$ a space of finite rational category. Then $f_{\#} \otimes \mathbb{Q}$ is zero in all even-degrees and $\operatorname{rank}\left(f_{\#} \otimes \mathbb{Q}\right) \leq \operatorname{cat}_{0}(X)$.

Proof. By assumption, $f_{\mathbb{Q}}: A_{\mathbb{Q}} \rightarrow X_{\mathbb{Q}}$ is a cyclic map. Hence $\left(f_{\mathbb{Q}}\right)_{\#}: \pi_{n}\left(A_{\mathbb{Q}}\right) \rightarrow$ $\pi_{n}\left(X_{\mathbb{Q}}\right)$ has image in $G_{n}\left(A_{\mathbb{Q}}\right)$ by Lemma 2.2 . Since $\left(f_{\mathbb{Q}}\right)_{\#}$ is identified with $f_{\#} \otimes \mathbb{Q}$, both assertions now follow by [FH82, Th.III].

We also have the following result that extends the first assertion of Proposition 3.5, and gives a further restriction on the rank of the homomorphism induced on rational homotopy groups by a cyclic map.

Recall that we defined the rational spherical homology of $X$ to be $S H_{n}(X ; \mathbb{Q})=$ $h_{\mathbb{Q}}\left(\pi_{n}(X) \otimes \mathbb{Q}\right)$ for each $n$, where $h_{\mathbb{Q}}: \pi_{*}(X) \otimes \mathbb{Q} \rightarrow H_{*}(X ; \mathbb{Q})$ is the rationalized Hurewicz homomorphism. The vector space dual of $h_{\mathbb{Q}}$ gives a homomor$\operatorname{phism}\left(h_{\mathbb{Q}}\right)^{*}: H^{*}(X ; \mathbb{Q}) \rightarrow \operatorname{Hom}\left(\pi_{*}(X) \otimes \mathbb{Q}, \mathbb{Q}\right)$. The subspace of $H^{*}(X ; \mathbb{Q})$ dual to $S H_{*}(X ; \mathbb{Q})$ is referred to as the rational spherical cohomology of $X$, and is denoted by $S H^{*}(X ; \mathbb{Q})$. Notice that $\left(h_{\mathbb{Q}}\right)^{*}$ restricts to an injection from $S H^{n}(X ; \mathbb{Q})$ into $\operatorname{Hom}\left(\pi_{n}(X) \otimes \mathbb{Q}, \mathbb{Q}\right)$. Now suppose that $\left(\mathcal{M}_{X}, d_{X}\right)$ is the minimal model of $X$. Since the differential $d_{X}$ is decomposable, it induces the trivial differential on the module of indecomposables $Q\left(\mathcal{M}_{X}\right)$. Therefore, by passing to cohomology from the quotient projection $\mathcal{M}_{X} \rightarrow Q\left(\mathcal{M}_{X}\right)$, we obtain a map $\zeta_{X}: H\left(\mathcal{M}_{X}\right) \rightarrow Q\left(\mathcal{M}_{X}\right)$. Under the natural identifications of $H\left(\mathcal{M}_{X}\right)$ with $H^{*}(X ; \mathbb{Q})$ and $Q\left(\mathcal{M}_{X}\right)$ with $\operatorname{Hom}\left(\pi_{*}(X) \otimes \mathbb{Q}, \mathbb{Q}\right)$, the map $\zeta_{X}$ is naturally identified with the dual of the rationalized Hurewicz homomorphism $\left(h_{\mathbb{Q}}\right)^{*}$ [FHT01, p.173].

Theorem 3.6. Let $f: A \rightarrow X$ be a map whose rationalization factors through an $H$-space.

(1) $f_{\#} \otimes \mathbb{Q}$ is zero on $\operatorname{kernel}\left(h_{\mathbb{Q}}: \pi_{n}(A) \otimes \mathbb{Q} \rightarrow H_{n}(A ; \mathbb{Q})\right)$, for each $n$.

(2) If $X$ is a space of finite rational category, then $f_{\#} \otimes \mathbb{Q}$ is zero in all evendegrees.

Proof. Recall that $f_{\#} \otimes \mathbb{Q}$ is identified with the map induced by $\mathcal{M}_{f}: \mathcal{M}_{X} \rightarrow \mathcal{M}_{A}$ on the (quotient) modules of indecomposables. We observed in Proposition 3.1 that the minimal model $\mathcal{M}_{f}: \mathcal{M}_{X} \rightarrow \mathcal{M}_{A}$ has image in the cycles of $\mathcal{M}_{A}$. This means, in particular, that any indecomposable terms occurring in the image of $\mathcal{M}_{f}$ must be indecomposable cycles in $\mathcal{M}_{A}$. Now the vector space of indecomposable cycles in $\mathcal{M}_{A}$ is isomorphic to the rational spherical cohomology of $A$. Assertion (1) follows.

Now suppose that $\operatorname{cat}_{0}(X)<\infty$ and that, as in the proof of Theorem 3.2, we have a factorization $\mathcal{M}_{f} \sim \mathcal{M}_{\tilde{f}} \circ \mathcal{M}_{g}: \mathcal{M}_{X} \rightarrow \mathcal{M}_{A}$, for $\mathcal{M}_{g}: \mathcal{M}_{X} \rightarrow \mathcal{M}_{Y}$ with $Y$ an $H$-space. In that proof, we showed that $\mathcal{M}_{g}$ has image contained in $\mathcal{I}$, the ideal of $\mathcal{M}_{Y}$ generated by the odd-degree generators (we did not use any hypothesis on 
$A$ for that part of the argument). In particular, if $x$ is an even-degree generator of $\mathcal{M}_{X}$, then $\mathcal{M}_{g}(x)$ is decomposable in $\mathcal{M}_{Y}$. It follows that $g$, and hence $f$, induces zero on all even-degree rational homotopy groups. This shows assertion (2).

Thus we can add the following to Proposition 3.5:

Corollary 3.7. Let $f: A \rightarrow X$ be a rationally cyclic map with $X$ a space of finite rational category. Then $\operatorname{rank}\left(f_{\#} \otimes \mathbb{Q}\right) \leq \sum_{n \text { odd }} \operatorname{dim}\left(S H_{n}(A ; \mathbb{Q})\right)$.

Finally, we turn to our generalization of Gottlieb's odd-degree result. We first observe a restriction on the homomorphism induced on rational cohomology by maps which factor through an $H$-space.

Theorem 3.8. Suppose $X$ has finite rational category. If $f: A \rightarrow X$ is a map whose rationalization factors through an $H$-space, then $H^{*}(f)\left(H^{\text {even }}(X ; \mathbb{Q})\right) \subseteq H^{+}(A ; \mathbb{Q})$. $H^{+}(A ; \mathbb{Q})$.

Proof. We show the minimal model counterpart of the assertion. It follows directly from Proposition 3.1 that the image of any cohomology class of $H^{*}\left(\mathcal{M}_{X}\right)$ that is represented by a decomposable cycle in $\mathcal{M}_{X}$ is contained in $H^{+}\left(\mathcal{M}_{A}\right) \cdot H^{+}\left(\mathcal{M}_{A}\right)$. To handle the case in which a cohomology class is represented by an indecomposable cycle, we use a fact established in the proof of Theorem 3.2. As in that proof, suppose we have a factorization $\mathcal{M}_{f} \sim \mathcal{M}_{\tilde{f}} \circ \mathcal{M}_{g}: \mathcal{M}_{X} \rightarrow \mathcal{M}_{A}$, where $\mathcal{M}_{g}: \mathcal{M}_{X} \rightarrow$ $\mathcal{M}_{Y}$ for $Y$ an $H$-space. Let $\mathcal{I}$ denote the ideal of $\mathcal{M}_{Y}$ generated by the odd-degree generators. Then we showed in Theorem 3.2 that $\mathcal{M}_{g}$ has image contained in $\mathcal{I}$. From this it follows that the image under $\mathcal{M}_{g}$ of any even degree generator is decomposable in the cycles of $\mathcal{M}_{A}$. Consequently, we have $H\left(\mathcal{M}_{f}\right)\left(H^{\text {even }}\left(\mathcal{M}_{X}\right)\right) \subseteq$ $H^{+}\left(\mathcal{M}_{A}\right) \cdot H^{+}\left(\mathcal{M}_{A}\right)$.

We do not obtain a restriction comparable to that of Theorem 3.8 for odd-degree cohomology. This is because any cohomology class of $H^{2 n+1}(A ; \mathbb{Q})$ is in the image of a homomorphism induced by a map $f: A \rightarrow K(\mathbb{Q}, 2 n+1)$, and such a map is a cyclic map.

In the following result, we do not assume that $X$ has finite rational category. Recall that $S H_{*}(f): S H_{*}(A, \mathbb{Q}) \rightarrow S H_{*}(X, \mathbb{Q})$ denotes the map induced on rational spherical homology by $f: A \rightarrow X$.

Theorem 3.9. Let $f: A \rightarrow X$ be a rationally cyclic map. If $S H_{*}(f) \neq 0$ then $X$ decomposes up to rational homotopy type as $X \simeq_{\mathbb{Q}} X^{\prime} \times K(\mathbb{Q}, n)$ for some simply connected space $X^{\prime}$.

Proof. The hypothesis $S H_{*}(f) \neq 0$ translates into the following: There is some $\alpha \in \pi_{n}(A) \otimes \mathbb{Q}$ whose image under the composition $H_{*}(f) \circ h_{\mathbb{Q}}: \pi_{n}(A) \otimes \mathbb{Q} \rightarrow$ $H_{\mathrm{n}}(X ; \mathbb{Q})$ is non-zero. Therefore, $f_{\#}(\alpha) \in \pi_{n}(X) \otimes \mathbb{Q}$ gives a non-zero spherical element $h_{\mathbb{Q}}\left(f_{\#}(\alpha)\right) \in S H_{\mathrm{n}}(X ; \mathbb{Q})$. But since $f$ is rationally cyclic, we have $f_{\#}(\alpha) \in$ $G_{n}(X) \otimes \mathbb{Q}$, with $h_{\mathbb{Q}}\left(f_{\#}(\alpha)\right) \neq 0$. By a theorem of Oprea (see [Hal88, Lem.1.1]), this implies the splitting $X \simeq_{\mathbb{Q}} X^{\prime} \times S^{2 n+1}$.

Note that Theorem 3.9 can also be interpreted as a restriction on the homomorphism induced on rational cohomology by a cyclic map: A cyclic map into a space not decomposable as above must have zero image in rational spherical cohomology. We are unsure whether or not the homomorphism induced on rational cohomology by a cyclic map may contain indecomposable terms in its image without incurring the splitting of Theorem 3.9. 
Corollary 3.10. Suppose $X$ has finite dimensional rational homology and that $f: A \rightarrow X$ is a rationally cyclic map. If $\chi(X) \neq 0$, then $S H_{*}(f)=0$.

Proof. From Theorem 3.8, the image of $H^{*}(f)$ in even degrees is decomposable, and hence non-spherical. Thus $S H_{*}(f)$ is zero in even degrees. Since $\chi(X) \neq 0$, the splitting of Theorem 3.9 cannot occur with $n$ odd-recall that $K(\mathbb{Q}, 2 n+1) \simeq_{\mathbb{Q}}$ $S^{2 n+1}$. Therefore, by that result, $S H_{*}(f)$ is zero in odd-degrees, as well.

In particular, we see that under the hypotheses of Corollary 3.10, the homomorphism induced by $f$ on rational homology is zero if all homology of $H_{*}(A ; \mathbb{Q})$ is spherical. Thus, the corollary, or better, Theorem 3.9, gives a satisfactory generalization to cyclic maps of Gottlieb's result concerning odd-degree Gottlieb groups.

From the above results, we can also extend the main result of [Lim82], at least in the simply connected case. In the original, very strong hypotheses are placed on $X$, namely that both $H_{*}(X)$ and $\pi_{*}(X)$ are finitely generated. If, in addition, we have $\chi(X) \neq 0$ and $A$ a co- $H$-space, then it is shown that a cyclic map $f: A \rightarrow X$ obtains $\Sigma f$ an element of finite order in $[\Sigma A, \Sigma X]$.

Recall that a rational co-H-space is a space $A$ such that $A_{\mathbb{Q}}$ is a co- $H$-space. Recall that a (rationally) elliptic space is one whose rational homology and rational homotopy are both finite dimensional. A result of Halperin [Hal77] states that the Euler characteristic of a rationally elliptic space is non-negative. Further, that in the case of positive Euler characteristic, that is, in the non-zero case, the rational cohomology is zero in odd degrees. For brevity, we refer to an elliptic space with positive Euler characteristic as an $F_{0}$-space. There are many interesting examples of such spaces, including even dimensional spheres, complex projective spaces, and "maximal rank pair" homogeneous spaces $G / H$. Products of $F_{0}$-spaces, and more generally many total spaces of fibrations in which base and fibre are $F_{0}$-spaces, are again $F_{0}$-spaces.

We can relax Lim's hypotheses as follows:

Corollary 3.11 (cf. [Lim82, Th.5.2]). Let $A$ be a rational co-H-space with finite dimensional rational homology and let $X$ be an $F_{0}$-space. Then any rationally cyclic map $f: A \rightarrow X$ has rationally trivial suspension, $\Sigma f \simeq_{\mathbb{Q}} *: \Sigma A \rightarrow \Sigma X$. If $A$ is assumed finite dimensional, then the subgroup of cyclic maps $G(\Sigma A, \Sigma X) \subseteq$ $[\Sigma A, \Sigma X]$ is a finite group.

Proof. Since cup products in $\widetilde{H}^{*}(A ; \mathbb{Q})$ are trivial and $H^{*}(X, \mathbb{Q})$ is evenly graded we can apply Theorem 3.8 to obtain $H^{*}(f)=0: \widetilde{H}^{*}(X ; \mathbb{Q}) \rightarrow \widetilde{H}^{*}(A ; \mathbb{Q})$. It follows that $H_{*}(\Sigma f)=0: \widetilde{H}_{*}(\Sigma A ; \mathbb{Q}) \rightarrow \widetilde{H}_{*}(\Sigma X ; \mathbb{Q})$, and hence that $\Sigma f \simeq_{\mathbb{Q}} *$. The assertion about finiteness follows from Lemma 2.6.

\section{EXAMPLES}

We give several examples of rationally cyclic maps. Our first examples illustrate that most results from the previous section are sharp. We then give some complete calculations of the set $G\left(A_{\mathbb{Q}}, X_{\mathbb{Q}}\right)$.

Since our results tend towards restricting the possibilities for rationally cyclic maps, we begin with an example that indicates rich possibilities for such remain. Of course, since any map into an odd-dimensional sphere and any rational Gottlieb element are rationally cyclic maps, we already have many examples. 
Example 4.1. Consider the map $f: S^{3} \times S^{4} \rightarrow S^{4}$, obtained by composing the quotient map $q: S^{3} \times S^{4} \rightarrow S^{7}$ with the Hopf map $\eta: S^{7} \rightarrow S^{4}$. It is well-known that $f$ is not rationally trivial, although it induces the trivial homomorphism on both rational homotopy and rational cohomology. Since $S^{7}$ is an $H$-space, $f$ itself, and not just its rationalization, factors through an $H$-space. Actually, the rationalization of $\eta$ is a rational Gottlieb element in $G_{7}\left(S^{4}\right) \otimes \mathbb{Q} \cong \pi_{7}\left(S^{4}\right) \otimes \mathbb{Q}$, and so $f$ is a rationally cyclic map by Theorem 2.1 . This shows that we need $H^{\text {odd }}(A ; \mathbb{Q})=0$ in Theorem 3.2, even though the map may be trivial on cohomology. This example suggests many others of the form $A \rightarrow S^{n} \rightarrow X$, with the second map a rational Gottlieb element.

Example 4.2. The image of $H^{*}(f)$ need not be decomposable in odd degrees, as we now illustrate. Take $A=S^{2} \vee S^{2} \cup_{\alpha} e^{5}$ with the 5 -cell attached by the iterated Whitehead product $\alpha=\left[\iota_{1},\left[\iota_{1}, \iota_{2}\right]\right] \in \pi_{4}\left(S^{2} \vee S^{2}\right)$. Then all cup products in $H^{*}(A ; \mathbb{Q})$ are trivial, with $H^{5}(A ; \mathbb{Q}) \cong \mathbb{Q}$ non-spherical. Now the quotient map $q: A \rightarrow S^{5}$ is a map into a rational $H$-space, and is therefore a rationally cyclic map. In degree $5, H^{*}(f)$ has indecomposable, non-spherical image.

Example 4.3. Inclusion of the bottom cell $f: S^{2} \rightarrow \mathbb{C} P^{\infty}$ is a map into an $H$ space, and hence is a cyclic map. Evidently, we have $H^{\text {odd }}\left(S^{2} ; \mathbb{Q}\right)=0$ and yet $f$ is not rationally trivial - it is not trivial on rational cohomology, in fact. This shows that we need the hypothesis of finite rational category on $X$ in Theorem 3.2. It also shows that without $\operatorname{cat}_{0}(X)<\infty$, then a cyclic map $f: A \rightarrow X$ may have $f_{\#} \otimes \mathbb{Q}$ non-zero in even degrees. Indeed, here we have $G_{2}\left(\mathbb{C} P^{\infty}\right)=\pi_{2}\left(\mathbb{C} P^{\infty}\right)$. This example illustrates the splitting of Theorem 3.9 for the case $n=2$.

For some spaces $X$, including the $F_{0}$-spaces mentioned above, the rational Gottlieb groups of $X$ coincide with the odd rational homotopy groups of $X$. In certain cases, this may generalize to a bijection $G\left(A_{\mathbb{Q}}, X_{\mathbb{Q}}\right) \cong \bigoplus_{n \text { odd }} H^{n}\left(A ; \pi_{n}(X) \otimes \mathbb{Q}\right)$ for any space $A$. We now give some examples.

If $X$ is an odd dimensional sphere $S^{2 n+1}$ then $X_{\mathbb{Q}} \simeq K(\mathbb{Q}, 2 n+1)$ is an $H$ space and therefore $G\left(A_{\mathbb{Q}}, X_{\mathbb{Q}}\right)=\left[A_{\mathbb{Q}}, X_{\mathbb{Q}}\right]=H^{2 n+1}\left(A ; \pi_{2 n+1}\left(S^{2 n+1}\right) \otimes \mathbb{Q}\right)=$ $H^{2 n+1}(A ; \mathbb{Q})$. When $X=S^{2 n}$ the same result holds but the calculation is slightly more involved. The following example includes the cases in which $X$ is an even dimensional sphere or a complex projective space.

Example 4.4. Suppose $X$ has rational cohomology algebra a truncated polynomial algebra on a single generator of even degree $2 n$. If $H^{*}(X ; \mathbb{Q}) \cong \mathbb{Q}[x] /\left(x^{k+1}\right)$, then $G\left(A_{\mathbb{Q}}, X_{\mathbb{Q}}\right) \cong H^{2 n(k+1)-1}(A ; \mathbb{Q})$. We argue as follows: First, $X$ has minimal model $\Lambda(x, y)$ with $|x|=2 n,|y|=2 n(k+1)-1, d_{X}(x)=0$, and $d_{X}(y)=x^{k+1}$. Suppose $f: A \rightarrow X$ is a rationally cyclic map with minimal model $\mathcal{M}_{f}: \mathcal{M}_{X} \rightarrow \mathcal{M}_{A}$ given on generators by $\mathcal{M}_{f}(x)=a$ and $\mathcal{M}_{f}(y)=b$, for $a, b \in \mathcal{M}_{A}$. Now consider an affiliated map $F: A \times X \rightarrow X$ for $f$. Then by taking into account the relative degrees of $x$ and $y$, we can write its minimal model $\mathcal{M}_{F}: \mathcal{M}_{X} \rightarrow \mathcal{M}_{A} \otimes \mathcal{M}_{A}$ on generators as $\mathcal{M}_{F}(x)=x+a$ and $\mathcal{M}_{F}(y)=y+b+b_{1} x+\cdots+b_{k} x^{k}$, for $b_{i} \in \mathcal{M}_{A}$. Since $\mathcal{M}_{F}$ is a DG map, we can equate the terms that occur in $d_{A} \mathcal{M}_{F}(y)$ with those that occur in $\mathcal{M}_{F} d_{X}(y)=(x+a)^{k+1}$. Thus we find that $d_{A}\left(b_{k}\right)=(k+1) a$. Now define a DG homotopy $\Psi: \mathcal{M}_{X} \rightarrow \mathcal{M}_{A} \otimes \Lambda(t, d t)$ on generators by setting

$$
\Psi(x)=a(1-t)+\frac{1}{k+1} b_{k} d t
$$


and

$$
\Psi(y)=b-\frac{1}{k+1} b_{k} a^{k}+\frac{1}{k+1} b_{k} a^{k}(1-t)^{k+1} .
$$

This is easily checked to commute with the differentials, and so defines a DG homotopy that starts at $\mathcal{M}_{f}$ and ends at a map that is zero on the even-degree generator $x$. So far, we have argued that a cyclic map $f: A \rightarrow X$ has minimal model given on generators by $\mathcal{M}_{f}(x)=0$ and $\mathcal{M}_{f}(y)=b$, up to homotopy. For a map of this form, $b$ must be a cycle in $\mathcal{M}_{A}$. Furthermore, two such maps are DG homotopic exactly when the cycles that $y$ is sent to by each represent the same cohomology class of $H\left(\mathcal{M}_{A}\right)$. This latter assertion is easily justified using the approach of [AL95], for instance.

Example 4.5. Suppose $X=G / T$ where $G$ is a compact, connected Lie group and $T$ is a maximal torus. Then $G\left(A_{\mathbb{Q}}, X_{\mathbb{Q}}\right) \cong \bigoplus_{n \text { odd }} H^{n}\left(A ; \pi_{n}(X) \otimes \mathbb{Q}\right)$ for any space $A$. For in this case the minimal model of $X$ is of the form $\Lambda\left(x_{1}, \ldots, x_{n}, y_{1}, \ldots, y_{n}\right)$ with $\left|x_{i}\right|=2, d_{X}\left(x_{i}\right)=0$ and the $y_{i}$ of odd degree with $d_{X}\left(y_{i}\right)$ a decomposable polynomial in the $x_{i}$. The rational cohomology of $X$ is evenly graded and the cycles $x_{i}$ correspond to a space of generators. See [FHT01, Prop.15.16 and Ex.2,p.448] for justification of these assertions. By Theorem 3.8, if $f: A \rightarrow X$ is rationally cyclic, then $H^{*}(f)=0$ since $H^{2}(A ; \mathbb{Q})$ cannot contain non-zero decomposable terms. Thus the minimal model $\mathcal{M}_{f}: \mathcal{M}_{X} \rightarrow \mathcal{M}_{A}$ of $f$ satisfies $\mathcal{M}_{f}\left(x_{i}\right)=0$ and $\mathcal{M}_{f}\left(y_{i}\right)=\xi_{i}$ for cycles $\xi_{i} \in \mathcal{M}_{A}$ and the result follows as above.

\section{REFERENCES}

[AL95] M. Arkowitz and G. Lupton, On finiteness of subgroups of self-homotopy equivalences, Cech Centennial Conference, Contemp. Math., vol. 181, Amer. Math. Soc., 1995, pp. 125.

[FH82] Y. Félix and S. Halperin, Rational L.-S. category and its applications, Trans. Amer. Math. Soc. 273 (1982), no. 1, 1-38.

[FHT01] Y. Félix, S. Halperin, and J.-C. Thomas, Rational homotopy theory, Graduate Texts in Mathematics, vol. 205, Springer-Verlag, New York, 2001.

[Got69] D. H. Gottlieb, Evaluation subgroups of homotopy groups, Amer. J. Math. 91 (1969), 729-756.

[Hal77] S. Halperin, Finiteness in the minimal models of Sullivan, Trans. Amer. Math. Soc. 230 (1977), 173-199.

[Hal88] Torsion gaps in the homotopy of finite complexes, Topology 27 (1988), 367375.

[HMR75] P. Hilton, G. Mislin, and J. Roitberg, Localization of nilpotent groups and spaces, NorthHolland Publishing Co., Amsterdam, 1975, North-Holland Mathematics Studies, No. 15, Notas de Matemática, No. 55.

[Lan75] G. Lang, Localizations and evaluation subgroups, Proc. Amer. Math. Soc. 50 (1975), 489-494.

[Lim82] K. L. Lim, On cyclic maps, J. Austral. Math. Soc. 32 (1982), 349-357.

[Opr95] J. Oprea, Gottlieb groups, group actions, fixed points and rational homotopy, Lecture Notes Series, vol. 29, Seoul National University Research Institute of Mathematics Global Analysis Research Center, Seoul, 1995.

[Tan83] D. Tanré, Homotopie rationnelle: modèles de Chen, Quillen, Sullivan, Lecture Notes in Mathematics, vol. 1025, Springer-Verlag, Berlin, 1983.

[Var69] K. Varadarajan, Generalised Gottlieb groups, J. Indian Math. Soc. 33 (1969), 141-164.

Department of Mathematics, Cleveland State University, Cleveland OH 44115

E-mail address: G.Lupton@csuohio.edu

Department of Mathematics, Saint Joseph's University, Philadelphia, PA 19131

E-mail address: smith@sju.edu 